\title{
The Model of Oceanic Crust Expansion
}

\author{
Daoxiong Hu \\ Xibu Drilling Engineering Co. Ltd. of CNPC, Karamay, China \\ Email: hudaoxiong@cnpc.com.cn
}

Received 18 October 2014; revised 10 November 2014; accepted 8 December 2014

Copyright (C) 2014 by author and Scientific Research Publishing Inc.

This work is licensed under the Creative Commons Attribution International License (CC BY). http://creativecommons.org/licenses/by/4.0/

(c) (i) Open Access

\begin{abstract}
Oceanic crust expansion belt is the largest extensional structure on the earth. All the crustal movement theories have to face the phenomenon of oceanic crust expansion. The paper tries to interpret the phenomenon of oceanic crust expansion by the volume change of the earth and the satellite data. The oceanic crust expansion is the result of the volume change of the earth. The volume change of the earth's mantle is the element causing the volume change of the earth; the state variation of the solid matters within the earth is the element leading to the volume increase of the earth's mantle; while the discharge of liquid matters within the earth is the element leading to the volume decrease of the earth's mantle. The process of oceanic crust expansion can be divided into two phases-expansion and top mounting. The phase of expansion is the volume increase of the earth's mantle, that is, the state variation of the solid matters within the earth leading to the oceanic crust expansion. The phase of top mounting is the volume decrease of the earth's mantle; that is, the matters of the earth's mantle (new oceanic crust) are uplifted to be the oceanic crust matters under the action of buoyancy, which is one of the ways to discharge the matters within the earth. The expansion phase of the oceanic crust is the extensional movement period of the earth's crust. The top mounting phase of the oceanic crust is the compressive movement period of the earth's crust, that is, the subduction period of the oceanic crust. The speed of oceanic crust expansion is related to the state variation speed of the matters within the earth. The width of the oceanic crust expansion is related to the temperature of the seawater. The temperature of the hydrothermal flow in the oceanic crust expansion belt is related to the speed of oceanic crust expansion. The oceanic ridge terrain in the oceanic crust expansion is related to the thickness of the same density stratification above the earth's mantle.
\end{abstract}

\section{Keywords}

Oceanic Crust Expansion, Oceanic Crust Top Mounting

\section{Introduction}

Oceanic crust expansion is the manifestation pattern of crustal movement. In the era without satellite pictures, 
the model of oceanic crust expansion is interpreted by the volcano model (such as the thermal convection theory and the mantle plume theory), that is to say, the oceanic crust formed due to the gushing of magma after the tension fracture of the oceanic crust, especially the existence of oceanic ridge, leads to the misunderstanding towards the oceanic crust expansion.

The model of oceanic crust expansion put forward in the paper is based on the theory of the earth volume change. According to the data on the satellite pictures and the seismic data, the new model of oceanic crust expansion has been put forward. It is the first time to divide the oceanic crust expansion into two phases - expansion and top mounting, and also the first time to put forward that the width of oceanic crust expansion is related to the temperature of seawater and that the phenomena including oceanic ridge and hydrothermal flow can be interpreted by the model of expansion.

The crustal movement is mainly expressed as the extensional movement and compressive movement. The oceanic crust expansion belt is the largest extensional structure on the earth; in the meanwhile, it is also the matters discharging zone leading to compressive crustal movement.

The drive of crustal movement comes from the volume change of the earth. The volume change of the earth refers to the volume change of the earth's mantle. With the volume change of the earth's mantle, the volume of the earth's shape also changes. The solid earth's crust covering (floating on) the earth's mantle is expressed as the changes of the earth's superficial area. The changes of the earth's superficial area are the extensional or compressive crustal movement; therefore, it is the volume change of the earth that leads to the crustal movement [1].

The element leading to the volume increase of the earth's mantle is the state variation of the solid matters within the earth. The solid matters within the earth are mainly the crashing planetesimals and subduction oceanic crust. The modern seismic data verify that some melting massive solids have been discovered in the earth's mantle. If the solid matters melt to be the liquid matters, the state variation incremental quantity will be generated. The volume change coefficient is approximate to 0.097 [1], and the state variation incremental quantity will lead to the volume increase of the earth's mantle.

The element leading to the volume decrease of the earth's mantle is the discharge of the liquid matters within the earth. The discharging ways of matters include oceanic crust expansion, magmatic intrusion, volcanic eruption, and hydrothermal penetration and so on. The discharge of matters makes the matters of the earth's mantle to be the earth's crust matters, thus leading to the volume decrease of the earth's mantle.

The volume increase of the earth's mantle leads to the extensional movement of the earth's crust; while the volume decrease of the earth's mantle leads to the compressive movement of the earth's crust. The oceanic crust expansion is the main way of the enlarging of the earth's superficial area; while the oceanic crust subduction is the main way of the diminishing of the earth's superficial area. The extensional and compressive movements of the earth's crust are mainly expressed as the expansion and subduction of the oceanic crust, and secondarily expressed as the subsidence and uplift of the continental crust.

The oceanic crust expansion belt is the zone that the earth's superficial area increases, that is, constantly generating new oceanic crust. The way of generating new oceanic crust is the tension fracture of the expansion belt. The earth's mantle matters in the fracture generate new oceanic crusts.

\section{The Mode of Oceanic Crust Expansion}

The oceanic crust expansion belt is zone leading to the volume decrease of the earth's mantle, that is, the matters discharging zone. After the tension fracture of the expansion belt, the new oceanic crust formed by the earth's mantle matters will be top mounted to the cracked space. The decrease of the earth's mantle matters lead to the subduction of the oceanic crust.

The expansion of the oceanic crust can be divided into two phases—expansion and top mounting.

\subsection{Phase of Expansion}

The phase of expansion is the period of the extensional movement of the earth's crust, that is, the period that the earth's superficial area increases.

When the volume of the earth's mantle increases, the oceanic crust begins to expand. The speed of the oceanic crust expansion is determined by the state variation speed of the matters within the earth.

Volume increase of the earth's mantle leads to the oceanic crust expansion. The incremental quantity of the 
volume of the earth's mantle in the phase of expansion can be expressed in the following formula:

$$
V=(T v \times 0.097)-P v
$$

In the formula,

$V$ refers to the incremental quantity of the volume of the earth's mantle in the phase of expansion;

$T v$ refers to the state variant of the solid matters within the earth in the phase of expansion;

0.097 refers to the comprehensive volume changing coefficient of the earth's matters (1500 degrees);

$P v$ refers to the discharge quantity of other matters in the phase of expansion, including the magmatic intrusion, volcanic eruption, and hydrothermal penetration and so on.

According to the calculation of the earth's volume change, during the forming stage of the earth (from 1.8 billion years ago to the present), the state variation speed of the matters within the earth is 0.55 cubic kilometers per day. If the calculation is made according to the volume change coefficient of 0.097 , the proportion of $83 \%$ that the oceanic crust expansion accounts for in the discharge of matters, the length of the expansion belt stretching for 15,000 km, the thickness of the oceanic crust being $10 \mathrm{~km}$, the width of the oceanic crust expansion belt being $66 \mathrm{~km}$, the cycle to form a group of oceanic crusts is approximately 750 thousand years, and the expansion speed of oceanic crust is approximately $8.8 \mathrm{~cm}$ per year [1]. The expansion speed of the ocean crust observed in the modern era is approximate to $6 \mathrm{~cm}$ to $9 \mathrm{~cm}$ per year.

At this expansion speed, in the initial phase of the oceanic crust expansion, the plastic magma of the earth's mantle exposed at the bottom of the expansion fractures, cools rapidly under the action of the seawater. By taking the exposed points as the radius, the semicircular crystalline rock mass is formed, which is the new oceanic crust. See the following Figure 1.

In the figure, $\mathrm{A}$ is the initial phase of oceanic crust expansion; B is the late stage of the oceanic crust expansion; $\mathrm{C}$ is the cooling point of the oceanic crust matters; $\mathrm{D}$ is the endpoint that the old oceanic crust and the new oceanic crust contact with each other as well as the deformation point of the new oceanic crust; the distance from Point $C$ to Point $D$ is the cooling radius, or it is called the lithogenetic thickness of the new oceanic crust; and Density 3.3 and Density 3.6 are the minimum density and maximum density of this part, while the actual situation is the more detailed delamination according to density.

Oceanic crust expansion is a slow process, which is only $0.24 \mathrm{~mm}$ or so every day. In the aspect of strained condition, what have to undergo the tension fracture every day on the contact surface (from Point C to Point D) between the new oceanic crust and the old oceanic crust are just the new crystalline rocks, that is $0.24 \mathrm{~mm}$. In the aspect of friction condition, the distance from Point C to Point D is the distance of friction. Under the huge stress, the huge scratch in the same direction of expansion will form on the top surface of the new oceanic crust.

With the widening of the expansion belt, the new oceanic crust crystalline rock mass gradually becomes the massive rock body. See Figure 1 B. In the meanwhile, the high-density magma in the lower part of the new oceanic crust also becomes the plastic magma.

With the widening of the expansion belt, the lithogenetic new oceanic crust is like a carrying pole, which carries two old oceanic crusts. Due to that the oceanic crust is in total submergence, the diagenesis of new oceanic crust stops the magma of the earth's mantle from uplifting. Under the buoyancy influence of the high-density magma of the earth's mantle, it will generate two consequences for the new oceanic crust, one is that the old oceanic crusts on the two sides of the expansion belt uplift, thus forming the upheaval of the expansion belt, which is called the oceanic ridge; the other is that the new oceanic crust body bends under stress, thus entering the phase of top mounting.

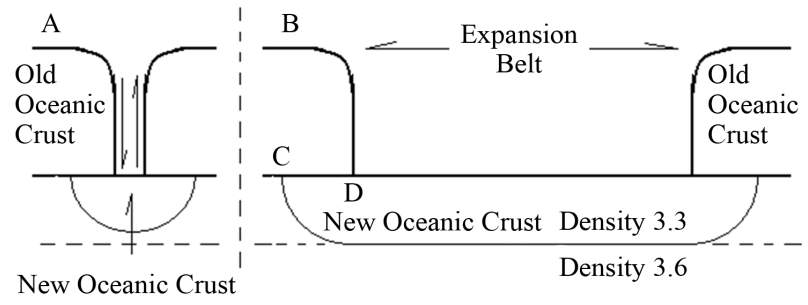

Figure 1. Schematic diagram of oceanic crust in expansion phase. 


\subsection{Phase of Top Mounting}

The phase of top mounting is the compressive movement period of the earth's crust, that is, the decrease of the earth's superficial area.

Top mounting is to uplift the new oceanic crust body and set the new oceanic crust body on the submergence line of the oceanic crust (to be paralleled with the old oceanic crust), which is named as top mounting in short. The drive of the top mounting is the buoyancy of the magma of the earth's mantle.

Whether the lithogenetic new oceanic crust can enter the phase of top mounting or not is determined by the elastic coefficient of the new oceanic crust body. The elastic coefficient is determined by the thickness of the new oceanic crust body and width of the expansion belt. The elastic coefficient is inversely proportional to the thickness of the new oceanic crust body and directly proportional to the thickness of the expansion belt.

The thickness of the new oceanic crust body is determined by the temperature of seawater. When the seawater contacts the new oceanic crust body, the temperature rises rapidly, the high-temperature seawater moves up and the low-temperature seawater moves down, thus forming the circulating cooling of seawater, which is also named as the hydrothermal flow of oceanic crust.

In the places that the temperature of the seawater is relatively low, the quantity of heat that is brought away is relatively large, and the thickness of the new oceanic crust body enlarges. The larger the thickness is, the smaller the elastic coefficient will be and the wider the expansion belt will be developed to be. According to satellite photograph, the widest part of the expansion belt is the Moens Oceanic Ridge in the north section of the Atlantic Ocean, which reaches $78 \mathrm{~km}$ or so [2]. See Figure 2.

In the part that the temperature of the seawater is relatively high, the quantity of heat that is brought away is relatively small, and the thickness of the new oceanic crust body is relatively small. The smaller the thickness is, the larger the elastic coefficient will be and the narrower the expansion belt will be developed to be. According to satellite photograph, the width of the expansion belt adjacent to the Pacific Ocean is only $66 \mathrm{~km}$ or so [2]; therefore, it can be concluded that the width of the oceanic crust expansion is determined by the temperature of the seawater. See Figure 3.

The increase of the oceanic crust width is to increase the elastic coefficient of the new oceanic crust body. The increase of the elastic coefficient is to increase the deformation space of the new oceanic crust body.

The phase of top mounting is under the buoyancy influence of the earth's mantle. When the elastic coefficient of the new oceanic crust body is met, the two ends of the new oceanic crust body will be mounted up to the bending shape, and the new oceanic crust body will like the piston, uplifting from the bottom surface of the old oceanic crust to the top surface of the old oceanic crust. See Figure 4.

In the above figure, the top surface of the oceanic crust is not only the end point of the top mounting of the new oceanic crust body, but also the beginning point that the oceanic crust starts to expand once again. While the new oceanic crust undergoes the top mounting, the relatively high-density plastic magma will also become one part of the new oceanic crust. The seismic data verify that the inside of the oceanic crust body is the pattern of delamination according to density.

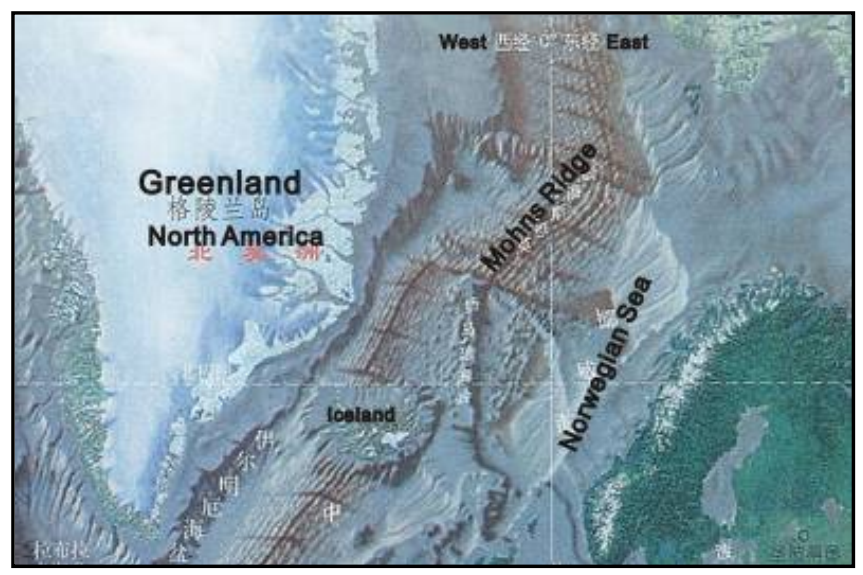

Figure 2. Oceanic crust expansion schematic diagram of Moens Oceanic Ridge. 


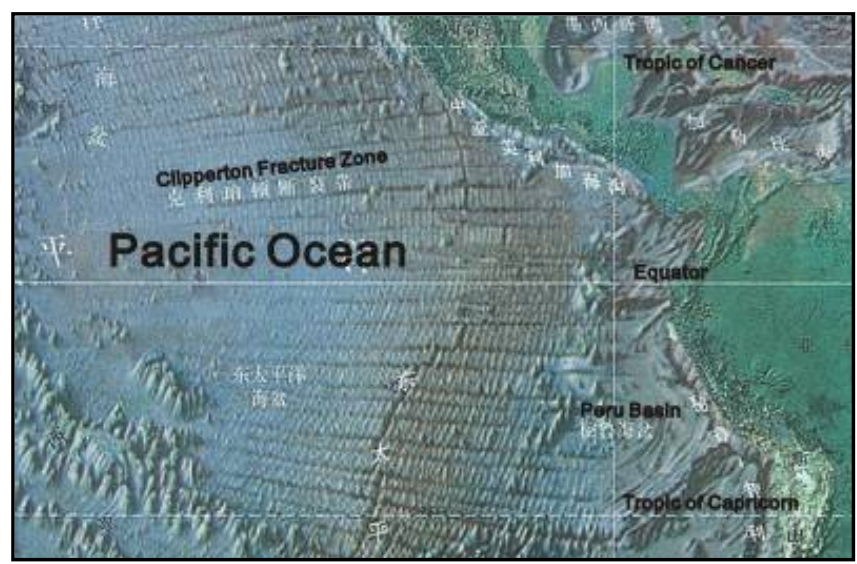

Figure 3. Oceanic crust expansion schematic diagram of Pacific Ocean near the equator.

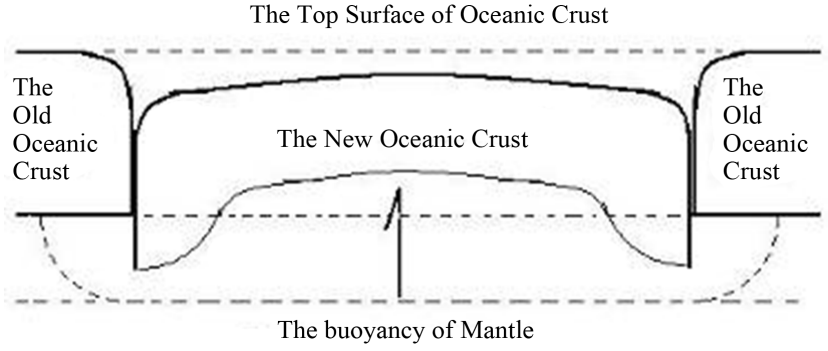

Figure 4. Schematic diagram of the top mounting phase of the oceanic crust.

When the width of the expansion belt reaches the degree that the new oceanic crust is able to deform, the expansion of the oceanic crust stops. When the top mounting begins, the subduction of the oceanic crust begins.

In the phase of top mounting, the new oceanic crust body has been a super-thick (8 km to $12 \mathrm{~km}$ ) rock mass. While entering the expansion belt (space of top mounting), the volume of the earth's mantle begins to decrease and the oceanic crust bears the extrusion force. Due to the support of the new oceanic crust body, the width of the expansion belt is relatively stable. The phase of top mounting leads to the volume decrease of the earth's mantle while the formed extrusion force is delivered to the subduction belt of the oceanic crust.

In the phase of top mounting, from the distribution of the expansion belt, due to the cutting of the transform fault, the time and process of the top mounting is not concurrent and it may be the sectional top mounting. The volume change of the earth's mantle in the phase of top mounting can be expressed in the following formula:

$$
D v=(D v 1-Z v)+T v+F v
$$

In the formula:

$D v$ : the volume of the earth's mantle after top mounting;

$D v 1$ : the volume of the earth's mantle before top mounting;

$Z v$ : the volume of the oceanic crust during top mounting;

$T v$ : the state variation incremental quantity during the phase of top mounting;

$F v$ : the volume of the subduction oceanic crust entering the earth's mantle.

From Formula (2), it can be seen that in the phase of top mounting, due to the top mounting of the oceanic crust, the volume of the earth's mantle decreases; however, the state variation incremental quantity and the subduction of the oceanic crust supplement the volume of the earth's mantle in time. The consequence in the phase of the top mounting is that the new oceanic crust moves upward to the expansion belt, that is, earth's mantle matters become the oceanic crust matters; while the old oceanic crust moves downward to the subduction belt, that is, the oceanic crust matters become the earth's mantle matters.

The expansion model of the oceanic crust depends on two links-expansion and subduction to fulfill the cir- 
culation of materials in the upper side of the earth's mantle. The expansion belt and the subduction belt of the oceanic crust are the two windows in the circulation chain. The expansion belt is the window to increase the earth's superficial area; while the subduction belt is the window to decrease the earth's superficial area. The oceanic crust is like the circulation chain in the surface layer of the earth. From the expansion belt of the subduction belt, during the process of circulation, the extensional movement (the volume increase of the earth's mantle) has been revealed and the compressive movement of the earth's crust has been promoted (volume decrease of the earth's mantle).

From the beginning of expansion to the end of the top mounting, it is a process from the volume increase of the earth's mantle to the volume decrease of the earth's mantle. It is the expansion model of the oceanic crust and also a cycle of the volume change of the earth.

The phase of expansion is the volume increase period of the earth's mantle. The element leading to the volume increase of the earth's mantle is the state variation of the solid matters within the earth. The melting speed is relatively low. The increase of the state variation incremental quantity and the volume of the earth's mantle is a slow process. The duration of the expansion phase of the oceanic crust is relatively long.

The phase of top mounting is the volume decrease period of the earth's mantle. The element leading to the volume decrease of the earth's mantle is the discharge of the earth's mantle matters, that is, the new oceanic crust formed by the earth's mantle matters is top mounted and become the oceanic crust matters. During the phase of top mounting, the earth's mantle matters decrease; besides, the decreased quantity is larger than the incremental quantity of the state variation of the solid matters within the earth during the same period. The speed of top mounting is related to the density of the upper layer of the earth's mantle below the expansion belt. The duration of the top mounting phase of the oceanic crust is relatively short.

The time difference between the expansion phase and the top mounting phase determines the pattern of the crustal movement, which is expressed as that the force bearing points of the extensional movement are dispersed and gentle; while the force bearing points of the compressive movement are concentrated and intense.

\section{Related Concepts of Oceanic Crust Expansion}

The model of the oceanic crust expansion will lead to some relevant geological phenomena, and the following is the detailed description:

\subsection{Oceanic Ridge}

Oceanic ridge is the visual name of the expansion belt. As for the expansion belt under expansion, its altitude is larger than that of the oceanic crusts on the two sides. In terrain, it is like the backbone or mountain ridge; therefore it is named as the oceanic ridge.

The element that forms the terrain of the oceanic ridge is that the thickness of the same density stratification under the expansion belt and on the top surface of the earth's mantle is smaller than that of the two wings of the expansion belt. Under the buoyancy influence of the high-density plastic magma on the top surface of the earth's mantle, the terrain of the oceanic ridge forms (See Figure 5).

The element leading to that the thickness of the same density stratification of the expansion belt is smaller than that of its two wings, is that the plastic magma matters forming the new oceanic crust. According to the principle of density stratification, the light matters below the expansion belt are top mounted and form the new oceanic crust. The matters with the same density of the two wings will definitely be migrated to the expansion belt. The light matters below the oceanic crust moves from the two wings to the expansion parts in the direction opposite to moving direction of the oceanic crust expansion and thermal convection theory (See a in Figure 5).

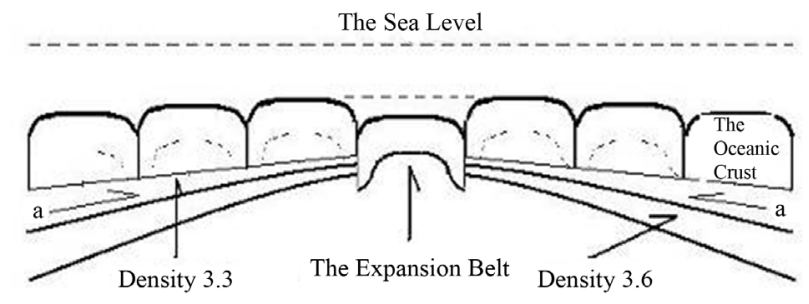

Figure 5. Schematic diagram of expansion belt (oceanic ridge). 
Due to that the matter below the oceanic crust is the plastic magma, the process to move towards the expansion belt is slow. During the continuous expansion of the expansion belt, before the restoration of the thickness of the light matters on the top surface of the earth's mantle, the terrain of the oceanic ridge will develop in succession. As for the expansion belt which stops expansion and the enclosed ocean basin, the terrain of the oceanic ridge will gradually disappear.

\subsection{Hydrothermal Flow of the Oceanic Crust}

The hydrothermal flow of the oceanic crust is the product of the oceanic crust expansion. As long as there is the oceanic crust expansion, there is the generation of the hydrothermal flow of the oceanic crust. During the process of the oceanic crust expansion, the seawater cools the earth's mantle matters, and forms the circulation in the expansion belt that the low-temperature seawater moves downward and the seawater with thermal energy moves upward, thus generating the hydrothermal flow of the oceanic crust.

The hydrothermal flow of the oceanic crust is just the circulating water that the tension fissure of the expansion belt cools the earth's mantle matters. As for the expansion belt under expansion, it is normal that the heat flux value of the seawater is higher than that of the other waters, which shall not be taken as the reference for the thermal convection theory and mantle plume theory. If there is no seawater for cooling, the oceanic crust expansion will be in another kind of model.

\subsection{Closure of Expansion Belt}

The closure of the expansion belt refers to that the expansion belt cannot go on expanding. The element leading to the closure of the expansion belt is that the oceanic crust volcanoes stop the oceanic crust from subduction and forms the field of stress within the oceanic crust, thus stopping the oceanic crust from expanding. The closure of the expansion belt means the closure of the ocean basin, such as the oceanic crust of the Indian Ocean which stops expanding. There are the closures of many ancient ocean basins, which are related to the oceanic crust volcanoes.

\subsection{Trend of the Expansion Belt}

The main trend of the expansion belt is approximately SN, which is vertical to the rotation direction of the earth. The element causing this consequence is the centrifugal force of the rotation, which generates the dispersive force in the rotation direction, that is, the tension paralleled to the rotation direction. The combination of the tension points vertical to the rotation direction of the earth, forms the expansion belt mainly in the SN trend. There are also some expansion belts which are not in the SN trend. When the volume changing energies of the earth are relatively small, most of them have stopped the expansion.

\subsection{Width of the Expansion Belt}

The width of the oceanic crust expansion belt is basically equivalent in the same waters, which suggests that under the influence of the seawater temperature, the thickness values of the oceanic crusts in the same waters are also basically the same. The oceanic expansion presents the regular width, which suggests that the state variation speed of the matters within the earth is balanced and the temperature of the seawater varies stably. In turn, it can analyze the seawater temperature during the formative period according to the width of the oceanic crust.

\section{Conclusions}

1) The fundamental of the model of the oceanic crust expansion is the volume change of the earth. The volume change of the earth is that the state variation of matters leads to the volume increase of the earth's mantle; the volume increase of the earth's mantle leads to the increase of the earth's superficial area; the increase of the earth's superficial area leads to the oceanic crust expansion; the oceanic crust expansion leads to the top mounting of the oceanic crust; the top mounting of the oceanic crust leads to the volume decrease of the earth's mantle; the volume decrease of the earth's mantle leads to the decrease of the earth's superficial area; the decrease of the earth's superficial area leads to the subduction of the oceanic crust; and the subduction of the oceanic crust leads to the increase of the state variation matters.

2) The volume change the earth is to establish the material circulation chain on the surface layer of the earth 
through the expansion and subduction of the oceanic crust. During the process of circulation, it continues to bring the light materials with state variation within the earth to the places below the continental crust from the places below the oceanic crust (expansion belt), thus forming the intrusive bodies of the continental crust and volcanoes, to make the continental crust thicker and thicker. During the circulation, the components of the continental crust become more and more complicated; while the components of the oceanic crust become simpler and simpler. During the circulation, the crustal movement is promoted. The continental crust conducts the multiphase and multi-direction superposition; while the oceanic crust makes unidirectional movement.

3) The oceanic crust expansion, in essence, is the increase of the earth's superficial area. It is the period that the earth's crust makes extensional movement. The top mounting of the oceanic crust leads to the volume decrease of the earth's mantle, thus maintaining the basic balance of the volume of the earth's mantle through the subduction of the oceanic crust. It is also the compressive movement period of the earth's crust. When the solid matters in the earth's mantle vary the state and the volume of the earth's mantle increase, it is impossible to pull back the oceanic crust which has been in the places below the continental crust by subduction. The part that the new oceanic crust which is top mounted in the expansion belt and the old oceanic crust combine with each other is the part most likely to undergo tension fracture and also the part that the oceanic crust begins to expand once again.

4) In the satellite photos, it can be seen that the expansion width in vicinity of the equator is smaller than that in vicinity of the polar region. In the aspect of spherical stress, this phenomenon is not supposed to occur. The main difference between the polar region and the equator is the temperature of the seawater. Temperature determines the crystallization thickness of the new oceanic crust, the crystallization thickness determines the elastic coefficient of the new oceanic crust, and the elastic coefficient determines the width of the new oceanic crust expansion; therefore, the width of the expansion belt in the north of the Atlantic Ocean with relatively low water temperature is larger than the width of the expansion belt in the Pacific equatorial regions. Water temperature determines the width of the expansion belt.

5) According to the seismic and drilling data of the oceanic crust, the density of the oceanic crust increases from the top to the bottom, that is, the pattern of delamination according to density, which suggests that before the diagenesis, the plastic magma below the oceanic crust is also in the state of delamination according to density. When the new oceanic crust is top mounted from the expansion belt, the low-density magma on the two ends of the expansion belt will definitely migrate to the expansion belt and fill in the bottom of the new oceanic crust, thus becoming the new oceanic crust matters of the next oceanic crust expansion. In this process, the flow direction of the magma is in the face-to-face direction, rather than the back-to-back direction advocated in the thermal convection theory to drive the oceanic crust expansion.

6) In the phenomenon of crustal movement, the oceanic crust expansion is a window. Through the oceanic crust expansion, it is observed that the volume of the earth's mantle is on the increase and the oceanic crust is in the period of extensional movement. With the top mounting of the oceanic crust and the earth's mantle becoming the oceanic crust matters, the volume of the earth's mantle is on the decrease and the oceanic crust is in the period of compressive movement. It is just because of the alternation of this extensional movement and compressive movement that forms the rich and colorful tectonic phenomena of the crustal movement. The oceanic crust expansion belt is the largest tectonic phenomenon on the earth and also the tectonic phenomenon able to reflect the dynamic mechanism of the crustal movement most. The model of the oceanic crust expansion is exactly the interpretation model of this phenomenon.

7) The volume change of the earth is the motivation theory of the crustal movement. All the motivation theories must interpret the expansion and subduction of the oceanic crust; and the model of the oceanic crust expansion must comply with the basic physical model.

The motive power of oceanic crust expansion is from Earth's volume change. Oceanic crust expansion does not result from thermal convection, but from mantle' volumetric increment. Additionally, the theory of thermal convection and mantle plume to explain oceanic crust expansion does violate the density principle of the movement of substances.

\section{References}

[1] Hu, D.X. (2009) Outline of the Earth's Volume Change. Xinjiang Science and Technology Publishing House.

[2] (2002) World Atlas by Countries. Star Map Publishing House. 
Scientific Research Publishing (SCIRP) is one of the largest Open Access journal publishers. It is currently publishing more than 200 open access, online, peer-reviewed journals covering a wide range of academic disciplines. SCIRP serves the worldwide academic communities and contributes to the progress and application of science with its publication.

Other selected journals from SCIRP are listed as below. Submit your manuscript to us via either submit@scirp.org or Online Submission Portal.
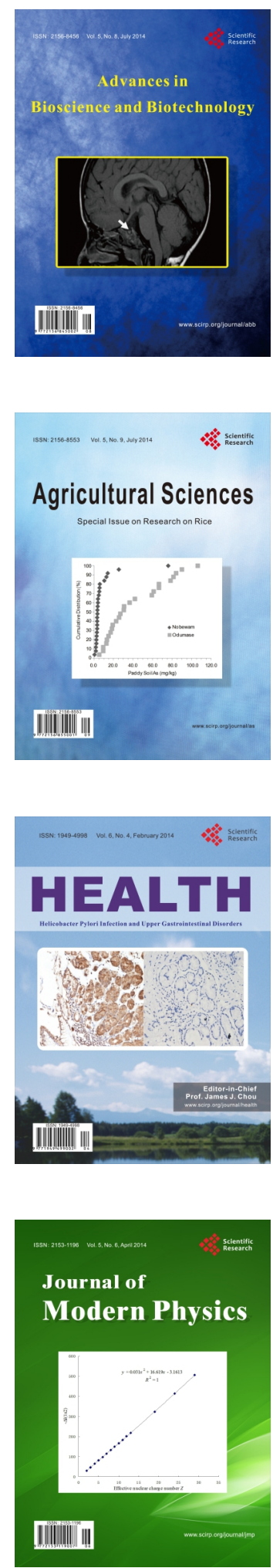
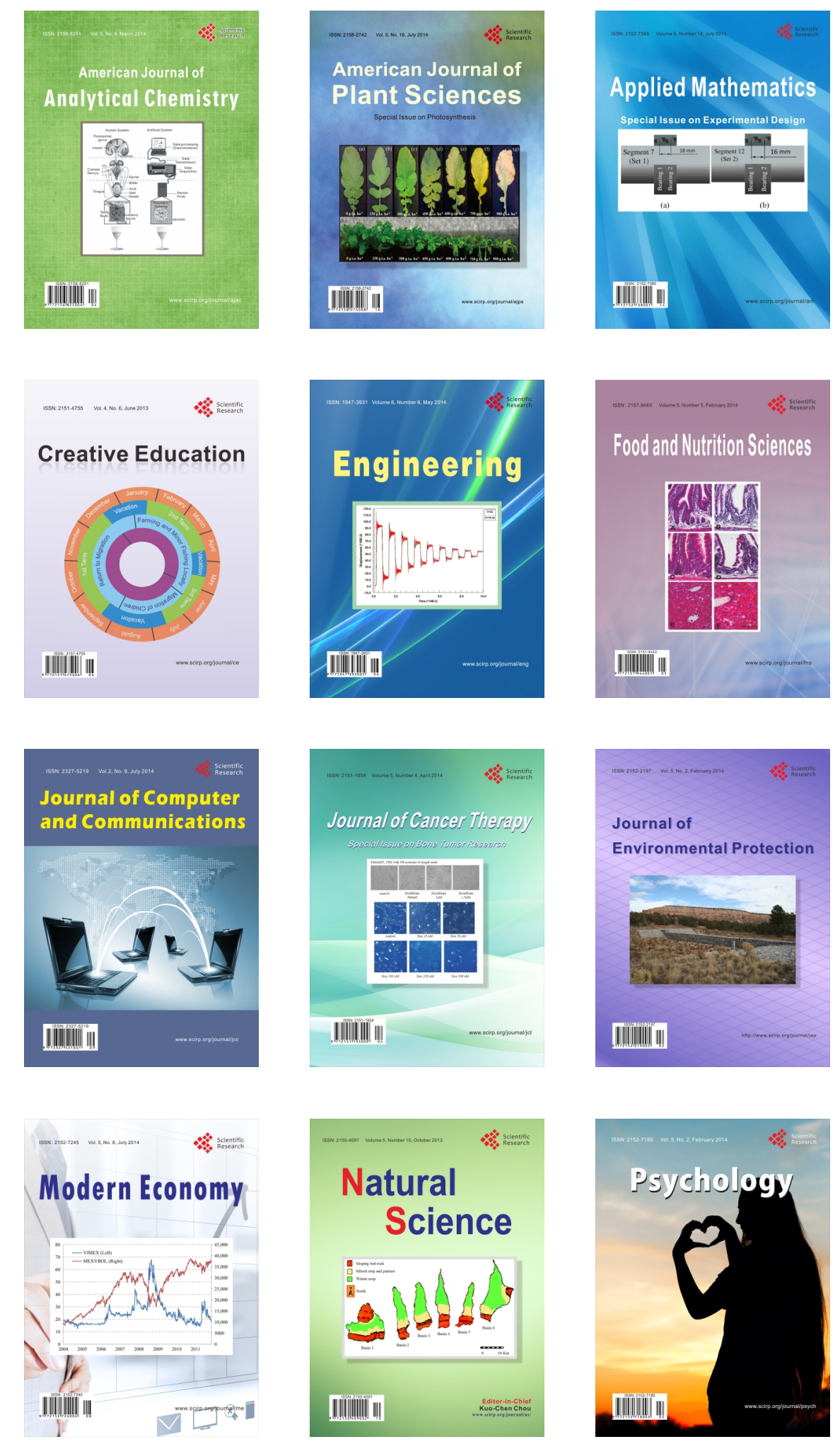